\title{
Molecular characterization and transcriptome profiling of expansin genes isolated from Calotropis procera fibers
}

\author{
Hafiza Masooma Naseer Cheema ${ }^{1} \cdot$ Aftab Bashir $^{2} \square \cdot$ Asia Khatoon $^{2} \cdot$ Nadia Iqbal $^{2} \cdot$ Yusuf Zafar $^{2}$ \\ Kauser A. Malik ${ }^{3}$ \\ 1 University of Agriculture, Faisalabad, Pakistan \\ 2 National Institute for Biotechnology and Genetic Engineering, Jhang Road Faisalabad, Pakistan \\ 3 Department of Biological Sciences, F.C. College University, Lahore, Pakistan \\ $\triangle$ Corresponding author: aftabbashir@nibge.org \\ Received February 21, 2010 / Accepted September 7, 2010 \\ Published online: November 15, 2010 \\ @ 2010 by Pontificia Universidad Católica de Valparaíso, Chile
}

\begin{abstract}
The Calotropis procera seed fibers provide an excellent model system to study the genes involved in fiber elongation, fineness and strength. Expansins constitute one of the important gene families involved in plant cell expansion and other cell wall modification processes. Four homologs of Expansin A gene i.e. CpEXPA1, CpEXPA2, CpEXPA3 and CpEXPA4 were isolated from the cDNA library obtained from fast growing Calotropis procera fibers. These homologs represented typical Expansin A family. Each of them had two conserved domains including GH45 like domain and the putative polysaccharide binding domain. The deduced amino acid sequences of the homologs indicated three conserved motifs: i) eight cysteine residues at $\mathrm{N}$-terminus, ii) four tryptophan residues at C-terminus and iii) a Histidine-Phenylalanine-Aspartate motif in the center of the sequence. The presence of $\mathrm{N}$-terminal signal peptide consisting of hydrophobic amino acids and a transmembrane region in all these expansin isoforms suggests their cotranslational insertion into the endoplasmic reticulum and then transportation to the cell wall by secretory pathway. The relative quantification of the four expansins in root, stem, fiber and leave tissues indicated that the transcripts of CpEXPA1, CpEXPA2, CpEXPA3 and CpEXPA4 are variably transcribed in these tissues. The lowest transcription of all the four Expansin $A$ isoforms was observed in elongating roots indicating that root tissue might be having specific expansins other than those confined to air grown organs.
\end{abstract}

Keywords: Calotropis procera, cDNA library, cotton fibers, EXPANSIN A, signal peptide, transcriptome profiling

\section{INTRODUCTION}

Calotropis procera belongs to the family of Asclepiadaceae (Mueen et al. 2005). The species is highly cross-pollinated and has 22 (2n) chromosomes (Raghavan, 1957; Eisikowitch, 1986). The plant is habituated to both sub-tropical and tropical climates. It is a deep rooted wild shrub ranging between 3-5 $\mathrm{m}$ and well acclimatized to salinity and draught. It produces $7-10 \mathrm{~cm}$ long spongy fruiting bodies bearing hundreds of seeds that are dispersed by winds upon maturity and fruit opening. The seeds have tufts of very fine and strong hollow fibers that range in length from $2.5-4.5 \mathrm{~cm}$ depending upon their position within the fruiting body. These fibers are botanically single celled seed trichomes. The $C$. procera bast fibers have been evaluated for their fiber characteristics in making cloth at a 1:1 ratio with cotton fiber (Varshney and Bhoi, 1988). The yarn has enough potential in natural fiber-reinforced composites and other industrial textile applications (Sakthivel et al. 2005). The floss may also substitute cotton wool for surgical or stuffing purposes (Eisikowitch, 1986). C. procera has generally been known since ancient times as a traditional medicinal plant (Oudhia, 1999a; Oudhia 1999b; Iqbal et al. 2005; Mueen et al. 2005). It has been used alone or in combination with other medicinal plants (Caius, 1986) to treat common diseases (Jain et al. 1996). Although, the plant has lived in close association with human beings for its beneficial traits, yet negligible information is available on the metabolomics, proteomics and genomics of this plant species. 
One of the important characteristics of this plant is the production of extra long hollow fibers associated with the seeds. This provides an excellent model system to study fiber expansion mechanism as the pathways involved in secondary cell wall deposition are naturally subtracted. Expansins are plant cell wall loosening proteins and have been proved to play a pivotal role in plant cell enlargement and expansion (Cosgrove, 2000; Cosgrove et al. 2002). Expansin gene family has versatile role in developmental processes like fruit softening, control of organ size, morphology, abscission, seed germination and pollen tube invasion in the grass stigma (Shcherban et al. 1995; Brummell et al. 1999a; Lee et al. 2003; Sabirzhanova et al. 2005). It has been proposed that expansin genes are involved in the slippage and rearrangement of the matrix polymers that coat the cellulosic microfibrils. The mechanism of cell wall loosening has been hypothesized to work by weakening the non-covalent binding between cell wall polysaccharides, thereby allowing the fibrils to slide over each other under the given cellular turgor pressure (McQueen and Cosgrove,1995; Marga et al. 2005).

Expansin comprises of four sub-families designated as $\alpha$-Expansin (EXPA), $\beta$-Expansin (EXPB), Expansin-like $A(E X L A)$ and Expansin-like $B(E X L B)$ (Kende et al. 2004). The EXPA and EXPB proteins cause cell wall loosening, whereas EXLA and EXLB proteins have not been fully characterized for their associated functions (McQueen-Mason et al. 1992; Cosgrove et al. 1997). The $\alpha$ and $\beta$ Expansin sub-families are found in all groups of land plants (Li et al. 2002; Schipper et al. 2002; Li et al. 2003). EXPAs disrupt the polysaccharide complexes like xyloglucan and pectin that link cellulosic microfibrils together (McQueen-Mason and Cosgrove, 1994; Marga et al. 2005). The pollen EXPBs (group-1 allergens) have a marked loosening action on grass cell walls, whereas, the EXPAsact on dicot cell walls only, therefore, it seems that these two families of expansin proteins target different component of cell wall (Cosgrove et al. 1997; Wu et al. 2001; Li et al. 2003; Kwasniewski and Szarejko, 2006). The coded proteins of both gene families have a number of conserved residues and characteristic motifs distributed throughout the length of the protein (Sampedro and Cosgrove, 2005).

C. procera seed fibers comprise of only the primary cell wall that is mainly composed of cellulose, lipids, proteins and polysaccharides like the primary cell wall of cotton fibers. Instead of four stage fiber development process, as reported in cotton, C. procera represents a three stage fiber development process that includes i) initiation, ii) elongation, and iii) maturation. The lack of secondary cell wall deposition in $C$. procera (stage iii in cotton) results in natural subtraction of the metabolic pathways involved in secondary cell wall deposition. The three stage fiber development feature has an excellent potential for the identification of genes directly involved in fiber elongation, strength and fineness. In cotton fibers, two expansin genes are reported to be specifically involved in loosening the cell wall of developing fibers (Harmer et al. 2002; Cheema et al. 2006). We identified four homologs of EXPAs expressed in the developing seed fibers of $C$. procera. The relative expression of the expansin homologs in various plant tissues indicated that none of those transcribed specifically in the fibers.

Table 1. Primer sequences used for real-time PCR analysis.

\begin{tabular}{|c|c|c|c|}
\hline Gene Name & Primer Name & Primer Sequence & Amplicon size \\
\hline СpEXРA1 & $\begin{array}{l}\text { CpExp1F BD } \\
\text { CpExp1R BD }\end{array}$ & $\begin{array}{l}\text { 5' TCTTCAATAATGGACTTAGC 3' } \\
\text { 5' GAGGAACATAGGCATAGC 3' }\end{array}$ & 200 \\
\hline СpЕХРА2 & $\begin{array}{l}\text { CpExp2F BD } \\
\text { CpExp2R BD }\end{array}$ & $\begin{array}{l}\text { 5' ATGCTTCTGGAACGATGG 3' } \\
\text { 5' TGGCGGTGACTATAATGC 3' }\end{array}$ & 186 \\
\hline СрЕХРАЗ & $\begin{array}{l}\text { CpExp3F BD } \\
\text { CpExp3R BD }\end{array}$ & $\begin{array}{l}\text { 5' TCTGTCCACCTAATCCTTCC 3' } \\
\text { 5' CCGTTGATTGTAACCTTATTCC 3' }\end{array}$ & 181 \\
\hline СpEXPA4 & $\begin{array}{l}\text { CpExp4F BD } \\
\text { CpExp4R BD }\end{array}$ & $\begin{array}{l}\text { 5' GCCACTCTTACTTCAACTTG 3' } \\
\text { 5' CTACACTCCGACCATCAC 3' }\end{array}$ & 195 \\
\hline $18 s r R N A$ & $\begin{array}{l}\text { RT18sF } \\
\text { Rt18sR }\end{array}$ & $\begin{array}{l}\text { 5' AAACGGCTACCACATCCAAG 3' } \\
\text { 5' CCTCCAATGGATCCTCGTTA 3' }\end{array}$ & 153 \\
\hline$\beta$-Tubulin & $\begin{array}{l}\text { RT } \beta-T u b F \\
\text { RT } \beta-T u b R\end{array}$ & $\begin{array}{l}\text { 5' CCATCTCCCAAGGTTTCTGA3' } \\
\text { 5' GCTTGAGGGTACGGAAACAG 3' }\end{array}$ & 139 \\
\hline
\end{tabular}




\section{MATERIALS AND METHODS}

\section{Plant material}

The plants of $C$. procera, growing in the NIBGE campus area, were selected for observing the flowering time, fruit development, maturation, size of fruiting body and fiber analysis. The flowers were tagged at 0 DPA (days post anthesis), indicated by flower opening, during March-September, 2007. The pollination was detected by the desiccation of petals and visualization of developing ovary. Increase in fruit size was measured till maturation and opening of fruit. Twenty five mature fruiting bodies were collected from different $C$. procera plants to collect $20-30 \mathrm{~g}$ of fiber per sample analysis. The plants of Gossypium hirsutum cultivar CIM-707 and G. barbadence cultivar Giza-88 were grown in the field during cotton season (May-September, 2007). Bolls from ten plants of each cotton species were picked and fiber was ginned. Fiber trait analysis was carried out by Fiber Technology Lab at CCRI, Multan. The fiber characteristics were determined in triplicate by High Volume Instrument (Uster HVI 900, Switzerland) using medium long and long calibration standards for CIM-707 and Giza-86 respectively. Fiber length was measured in millimeters $(\mathrm{mm})$. Micronaire was taken in mic $(\mu \mathrm{g} / \mathrm{inch})$ as an indicator of fiber fineness. The uniformity index was obtained by dividing the mean fiber length by the upper half mean length and expressing the ratio as percent. Bundle fiber strength was measured in $\mathrm{g} /$ tex. C. procera calibrator standards are not available and there is no previous report that describes the fiber analysis of this plant species. Therefore, the C. procera fiber characteristics were observed against cotton fiber standards.

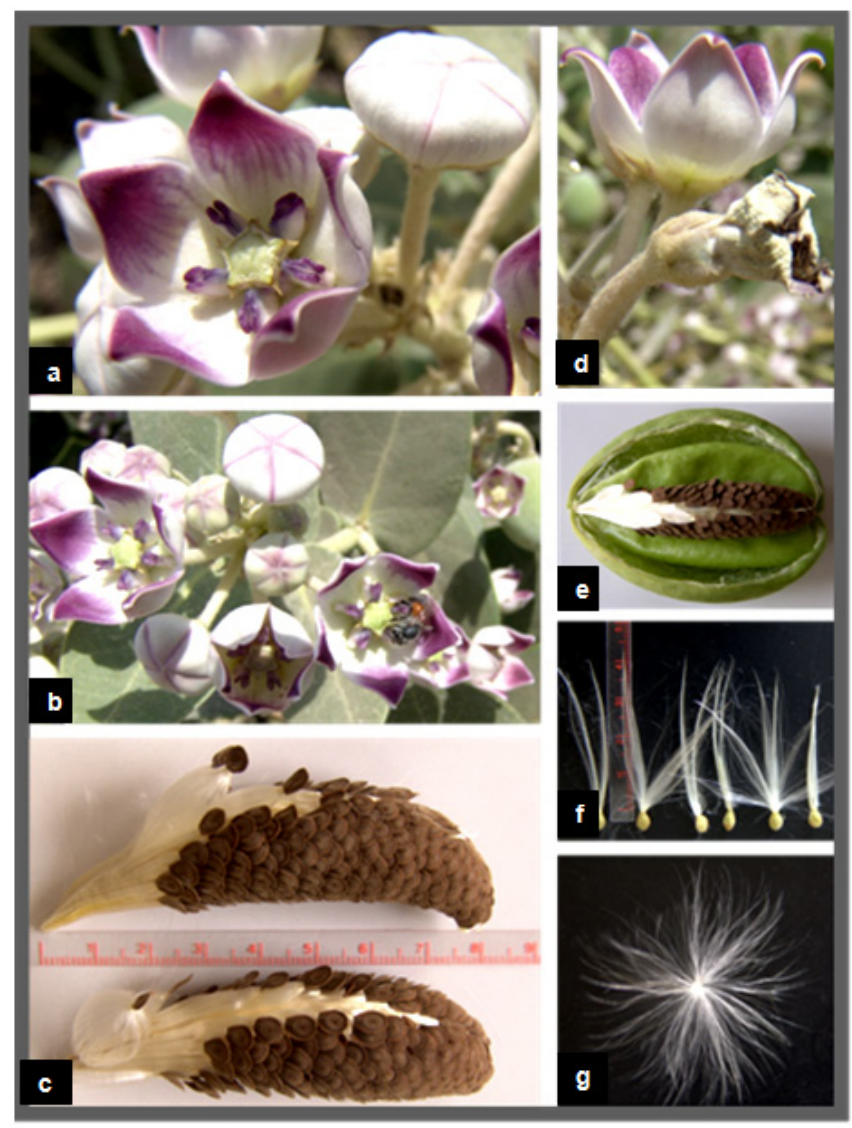

Fig. 1 Flower and fiber development stages of Calotropis procera. (a) Blossomed flower. (b) Insect as a source of cross pollination. (c) The cone bearing the seeds and fibers. The cone is about $8.5 \mathrm{~cm}$ in length. The end with fibers without seeds indicate attachment region on the stem and has shortest fibers $(2.5-3.0 \mathrm{~cm})$. The fibers on the other end are largest $(4-4.5 \mathrm{~cm}$ ). (d) Withering of the petals and initiation of fruit development. (e) Mature opened fruit indicating the outer integument, spongy layer, inner integument and the cone bearing the fibers. (f) The fiber length at the distal end region of the cone. $(\mathrm{g})$ Seed carrying the tuft of fibers and ready for seed dispersal. 


\section{Isolation of mRNA from $C$. procera fibers}

The fruits of $C$. procera were collected in liquid nitrogen at 10 DPA. The fibers were aseptically separated from the seeds. Total RNA was extracted using plant RNA purification reagent (Invitrogen, USA) according to the manufacturer instructions. The integrity and quality of the isolated RNA was checked by running the total RNA on $1 \%$ agarose gel. The mRNA isolation was carried out using oligo dT cellulose columns (MRC, USA). The purified mRNA was precipitated and dissolved in DEPC (diethyl polycarbonate) treated water.

\section{Construction of cDNA library}

The cDNA library of $C$. procera seed fibers was constructed using CloneMinor cDNA library construction kit (Invitrogen, USA). The manufacturer's instructions were strictly followed at each step using $5 \mu \mathrm{g}$ of mRNA at the cDNA synthesis step. The cDNA library was qualified by determining the transformation efficiency and the average size of inserts. The library was finally amplified and glycerol stocks were preserved at $-80^{\circ} \mathrm{C}$.

\section{Screening of cDNA library}

Serial dilutions of the fiber cDNA library were prepared and spread on LB medium having $50 \mu \mathrm{g} / \mathrm{ml}$ Kanamycin. The colony forming units (cfu) per $\mathrm{mL}$ of the library were determined. The library dilution to yield 40,000-50,000 cfu was spread on a $20 \times 20 \mathrm{~cm}$ plate. The colonies were lifted onto $20 \times 20 \mathrm{~cm}$ membrane (Hybond $\mathrm{N}+$, Fermentas) that was processed for colony hybridization. Cotton fiber specific expansin (accession number DQ023525) was used to probe this library for screening the expansin homologs present in the $C$. procera fiber. The probe was radiolabeled with $\alpha^{32} \mathrm{P} d A T P$ using hexalabel DNA labeling kit (Fermentas, USA). The hybridization was performed at $52^{\circ} \mathrm{C}$ (medium stringency) to pick all the possible expansin homologs from Calotropis fiber cDNA library.

\section{Sequencing the screened clones}

The plasmids from the expansin positive clones were isolated using plasmid miniprep kit (Fermentas). BigDye terminator cycle sequencing kit version 3.1 (Applied Biosystems, USA) was used for DNA sequencing on an $\mathrm{ABI} 310$ Genetic Analyzer. About $2 \mu \mathrm{g}$ of plasmid DNA was used per sequencing reaction. Initial DNA sequence at both ends was determined by using M13 forward and reverse primers. The complete nucleotide sequencing of each insert was accomplished by primer walking. The sequences were submitted in the GenBank and their accession numbers were obtained.

\section{Molecular analysis of the sequencing data using bioinformatics tools}

Amino acid sequence was derived for each expansin homolog using translate tool at http://www.expasy.ch. The amino acid sequences for all reported expansin genes were obtained from Swiss-Prot/TrEMBL.CLCbio combined workbench (CLCbio, Denmark) was used to align amino acid sequences and construct phylogenetic tree. The signal peptide and maximum cleavage site probability in the amino acid sequences was determined by SignalP 3.0 at http://www.cbs.dtu.dk/services/SignalP (Von Heijne, 1986; Bendtsen et al. 2004). The predicted amino acid sequences for EXPA1, EXPA2,

Table 2. Fiber characteristics of Calotropis procera as compared to cotton fiber.

\begin{tabular}{ccccc}
\hline Species & $\begin{array}{c}\text { Micronaire } \\
\text { (Mic) }\end{array}$ & $\begin{array}{c}\text { Fiber Length } \\
(\mathbf{m m})\end{array}$ & $\begin{array}{c}\text { Fiber Strength } \\
\mathbf{( g / t e x )}\end{array}$ & $\begin{array}{c}\text { Uniformity Index } \\
\mathbf{( \% )}\end{array}$ \\
C. procera & 2.09 & 42.0 & 29.5 & 81.6 \\
G. hirsutum & $4.5-4.9$ & $28-32$ & $28-32$ & $80-85$ \\
G. barbadence & $4.2-4.5$ & $32-35$ & $29-31$ & $80-85$ \\
\hline
\end{tabular}


EXPA3 and EXPA4 were compared using the multiple sequence alignment programs Kalign and Kalignvu, available at http://msa.cgb.ki.se/cgi-bin/msa.cgi (Lassmann and Sonnhammer, 2006). The amino acid sequence identity was calculated by the following formula: Identity $(\%)=100 \times$ number of identical residues in a pairwise alignment/length of the shortest sequence.

\section{Real-time PCR studies}

High quality total RNA was isolated from $100 \mathrm{mg}$ tissue samples, which included C. procera fibers, leaves, stem and roots. The total RNA was treated with DNAse I (Promega, USA) according to the manufacturer's instruction. The integrity of RNA was checked by electrophoresis on $1 \%$ agarose gel containing ethidium bromide. Relative quantification was carried out for real time PCR studies after normalization against unit mass as described before (Real-Time PCR applications guide, Bio-Rad Laboratories Inc. USA). The RNA samples were quantified on Ultrospec3100 (GE Healthcare, Europe) and equalized prior to cDNA synthesis. Revert Aid $\mathrm{H}^{-}$cDNA synthesis kit (Fermentas) was used for cDNA synthesis from 500 ng of equalized total RNA preps using oligo-dT (20 mer) primer according to the manufacturer's instruction. The equalization of different samples for cDNA content was performed both spectrophotometrically and by PCR amplification of 18SrRNA and $\beta$-tubulin genes. Beacon Designer 7 (Premier Biosoft International, USA) was used to design the primers on each expansin (Table 1). Multiple sets of primers were designed and used for validation on each expansin homolog. The primer pairs indicating amplification efficiency above $90 \%$ and a single amplification product, indicated by the melt curve, were selected for use in the relative quantification studies.

(a)

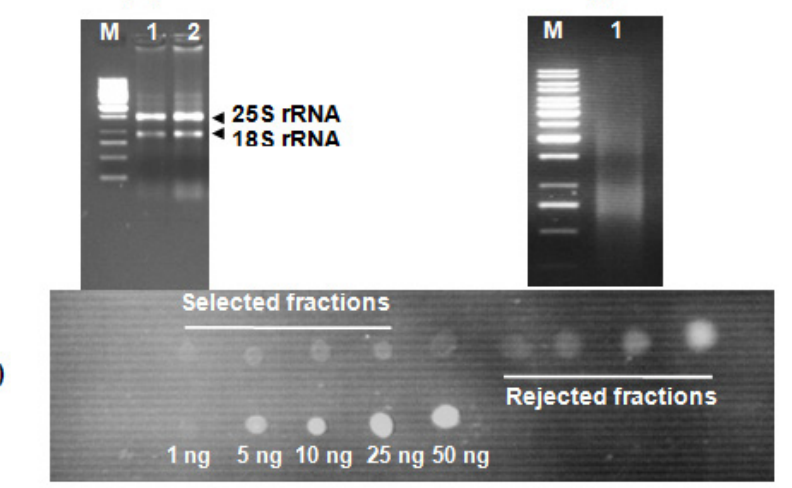

(d)

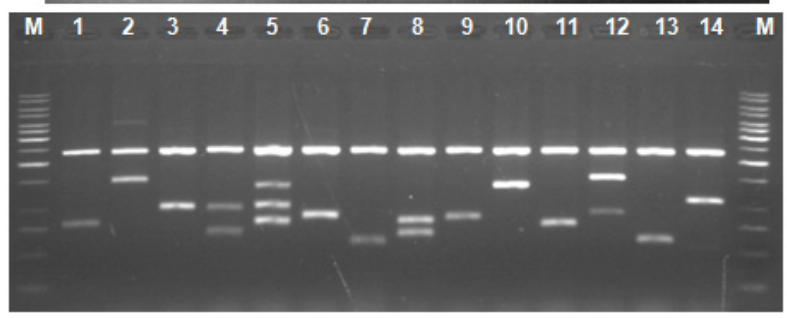

(e)

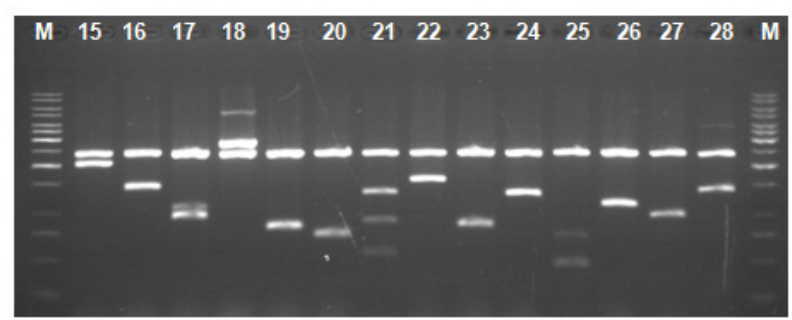

Fig. 2 Steps involved in constructing cDNA library and qualifying the library for different criteria. (a) $1 \mu \mathrm{L}$ of total RNA run on $1 \%$ agarose gel depicting the integrity of RNA represented by sharp $25 \mathrm{~S}$ and $18 \mathrm{~S}$ rRNA bands. (b) Agarose gel electrophoresis of $5 \mu \mathrm{L}$ of first strand cDNA showing the required range of cDNA sizes. (c) $1 \mu \mathrm{L}$ of each of first eleven collected fractions spotted on $1 \%$ agarose as compared to the spotted standard of known concentration. (d-e) Restriction digestion analysis of 28 randomly selected clones with Apal and EcoRV to determine percent recombination and average insert size. 
The real-time PCR mixture contained $1 \mu \mathrm{L}$ of diluted cDNA (corresponding to $10 \mathrm{ng}$ of starting amount of total RNA), $12.5 \mu \mathrm{L}$ of 2xSYBR Green Super Mix (BioRad, USA), and $25 \mathrm{ng} / \mu \mathrm{L}$ of each gene-specific primer in a final volume of $25 \mu \mathrm{L}$. The real-time PCR reactions were performed using 96 well plates sealed with optically clear sheets in iQ5 Cycler (BioRad). The reactions for amplifying CpEXPA1, CpEXPA2, CpEXPA3 and CpEXPA4 in various tissues were carried out in four replicates. The Ct value of root tissue sample was taken as calibrator for transcription profiling of expansin homologs in different tissues (root, stem, fiber and leaves). The relative fold expression was determined by unit mass method based on the ratio between test/calibrator $=2^{\Delta \mathrm{Ct}}$, where $\Delta \mathrm{Ct}=\mathrm{Ct}$ (calibrator) - Ct (test) and calibrator is the $\mathrm{Ct}$ of the roots sample for a particular expansin gene. Whereas, test indicates the $\mathrm{Ct}$ of a particular expansin homolog in tissues other than roots.

\section{RESULTS}

\section{C. procera fruit developmental studies}

The $C$. procera fruit size and number are dependent on the age and health of plant. The plant bears tufts of flowers at the distal end of newly branching shoots. The fruit number varies from 1-3 per flowering site (tuft of flowers). An average of 23 fruits per plant was observed during the fruiting season. The maximum fruit length varied from 7.5-9.5 cm depending upon the season. The maximum fruit length was attained in 20-25 days in early season, while this period prolonged in the late season. The fruit generally matured in 30-35 days (Figure 1 ).

\section{C. procera fiber characteristics}

The fiber length of mature fruits ranges between 2.5-4.5 cm depending upon its position in the fruit. Mature fiber characteristics including fiber length, fineness, uniformity/maturity and strength were better or equivalent to the finest grades of cotton fiber (Table 2).

\section{Identification of C. procera expansins}

The quality of total RNA, mRNA, and cDNA used for the construction of library was according to the recommended standards (Figure $2 a$, Figure $2 b$ and Figure $2 c$ ). After transforming the recombinants, the library was qualified for primary titer, average insert size and percent recombination. The titer of the cDNA library was $1.5 \times 106 \mathrm{cfu} / \mathrm{mL}$. Recombination efficiency was determined by the presence of the inserts in the vector. The CloneMiner cDNA library construction kit (Invitrogen) yielded 100\% recombination efficiency as indicated by the restriction analysis of 28 randomly selected primary clones (Figure $2 \mathrm{~d}$ and Figure $2 \mathrm{e}$ ). The average insert size of the library was $1.4 \mathrm{kbp}$ (Figure $2 \mathrm{~d}$ and Figure 2e). The primary, secondary and tertiary screening of cDNA library for expansin gene yielded fifty clones that were processed for DNA sequencing of the inserts. The analysis of the sequencing data indicated that four homologs of expansin gene family are expressed in the developing $C$. procera fibers. The

Table 3. Signal peptide and significant transmembrane regions in the deduced amino acid sequence of CpEXPA1, CpEXPA2, CpEXPA3 and CpEXPA4.

\begin{tabular}{ccccc}
\hline & $\begin{array}{c}\text { Cleavages site } \\
\text { probability }\end{array}$ & $\begin{array}{c}\text { Predicted signal } \\
\text { peptide length (a.a) }\end{array}$ & $\begin{array}{c}\text { Predicted cleavage } \\
\text { site in the amino acid } \\
\text { sequence }\end{array}$ & $\begin{array}{c}\text { Significant } \\
\text { transmembrane } \\
\text { segment (a.a) }\end{array}$ \\
CpEXPA1 & 0.963 & 22 & IQA - RI & $17-36(20)$ \\
CpEXPA2 & 0.993 & 26 & VHG - YY & $2-24(23)$ \\
CpEXPA3 & 0.992 & 24 & TLA - DY & $1-19(19)$ \\
CPEXPA4 & 0.858 & 17 & VHG - YG & $1-20(20)$ \\
\hline
\end{tabular}

Signal peptide was determined by applying Neural Network (NN) and Hidden Markov Model (HMM) trained on eukaryotes available at http://www.cbs.dtu.dk/services/SignalP/ and transmembrane segments were identified by TMpred available at http://www.ch.embnet.org/software/TMPRED. 
BLAST search, phylogenetic analysis, and the presence of conserved motifs suggested that they belong to Expansin $A$ gene family. According to the recommended nomenclature of expansin gene family (Kende et al. 2004), these genes were named as CpEXPA1, CpEXPA2, CpEXPA3 and CpEXPA4 (GenBank Accession no. EF434781, EF434782, EF434783 and EF434784, respectively). The alignment at amino acids level showed only $64.9 \%$ identity in the best aligned region between amino acids $40-240$ (Figure 3 ). The amino acid sequence identity among any of the two homologs ranged from $63.03 \%$ to $72.13 \%$ depicting a higher level of diversity at nucleotide level.

\section{Phylogenetic analysis}

The phylogenetic analysis of CpEXPA1, CpEXPA2, CpEXPA3 and CpEXPA4 showed the distribution of these homologs in different clades (Figure 4). The EXPA gene family has been distributed in 12 clades (Sampedro and Cosgrove, 2005). The expansin homologs were grouped in three of them i.e. clade I, III and IV. CpEXPA1 was grouped in clade IV, while CpEXPA2 and CpEXPA4 were observed in clade I. CpEXPA2 was closely associated with AtEXPA5, which is a representative of clade I under the same root, whereas, CPEXPA4 appeared under different root in the same clade. The clade III contained CpEXPA3 along with two fiber specific expansins of G. hirsutum i.e. GhEXPA1 and GhEXPA2.

\section{Analysis of signal peptides in expansin homologs}

The multiple alignment of expansin homologs and analysis for signal peptides at http://www.expasy.ch showed that CpEXPA1, CpEXPA2, CpEXPA3 and CpEXPA4 contained two domains (Figure 3). The $\mathrm{N}$-terminal domain comprised of the first 165 amino acids and contained the signal peptides. The remaining amino acids in the expansin homologs comprised the $\mathrm{C}$-terminal domain. The analysis for signal peptide (Predoter) and its cleavage (SignalP 3.0) indicated the targeting of expansins to endoplasmic reticulum (ER). The ER association of the expansin homologs indicated that all of them represent secretory proteins. The length of signal peptides was observed to be different for all the four homologs. Signal peptides of CpEXPA1, CpEXPA2, CpEXPA3 and CpEXPA4 were 22, 26, 24 and 17 amino acids long respectively. A transmembrane region was also detected at $\mathrm{N}$-terminus of all the expansin polypeptides (Table 3).

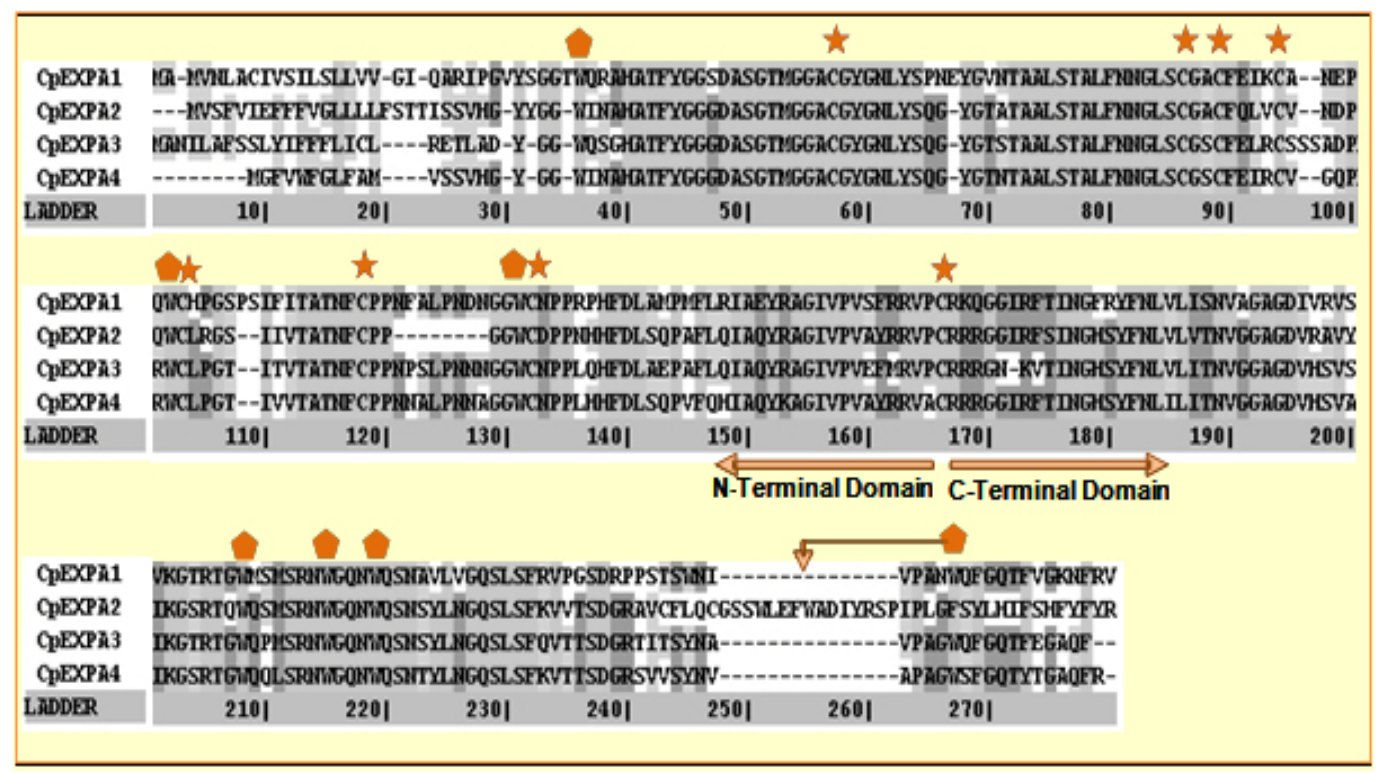

Fig. 3 Multiple sequence alignment of four homologs of CpEXPAs using Kalign and Kalignvu available at http://msa.cgb.ki.se/cgi-bin/msa.cgi. Ladder in the alignment is determined by the total number of aligned pairs divided by the maximum number of possible pairs. Shading over the amino acid sequence is based on the degree of conservation. First 165 amino acids comprise of $\mathrm{N}$-terminal domain having signal peptide of varying length, while the remaining is shown as C-terminal domain. Eight conserved cystein (C) residues and seven tryptophan (W) residues are indicated by stars and pentagonals respectively. 


\section{Sequence conservation}

Though the deduced amino acid sequences of four $C$. procera expansins had only $47.2 \%$ to $59 \%$ identity with each other (Table 1), they had many conserved motifs along the entire length. The amino acid sequence alignment showed eight conserved residues of cysteine $(C)$ at $N$-terminal and seven conserved residues of tryptophan $(\mathrm{W})$. The cysteine residues were associated with the $\mathrm{N}$-terminal domain, while tryptophan amino acid was distributed in both the $\mathrm{N}$-terminal and $\mathrm{C}$-terminal domain (Figure 3). The position of the last tryptophan in CpEXPA2 is not in line with the other CpEXPs due to an extension at the $\mathrm{C}$-terminal, which is not found in other $C p E X P A s$ sequences. A deletion of 8 amino acids was observed in CpEXPA2 between conserved domain TATNFCPP (amino acid sequence represented by single letter codon i.e. Threonine-Alanine-Threonine-Asparagine-PhenylalanineCysteine-Proline-Proline) and GGWC (Glycine-Glycine-Tryptophan-Cysteine) before the conserved motif "HFD" (Histidine-Phenylalanine-Aspartic acid), which is a part of the conserved GH45 active site. The CpEXPA1 indicated the addition of Glutamic acid (E) at position 67 and Proline-Serine (PS) at position 108. In CpEXPA3, the addition of two amino acids SS (Serine- Serine) is located at position 96. The signal peptides of all the four CpEXPs have low homology, especially in the first 40 amino acids, indicating the diversity with respect to their function (Figure 3).

\section{Transcript profiling of expansin homologs}

Relative expression studies for CpEXPA1, CpEXPA2, CpEXPA3 and CpEXPA4 revealed that none of these expansins are fiber specific, however, CpEXPA1 produced maximum transcripts in stem and fibers as compared to other tissues (Figure 5). The highest transcription of CpEXPA2 was observed in leaves along with the equivalent transcription level of CpEXPA4 while the transcripts of CpEXPA3 accumulated at the highest level in stem. The transcript level of CpEXPA1 and CpEXPA3 was observed to be almost the same in stem tissues.

\section{DISCUSSION}

Modification of cotton fiber traits for improving length, strength and finesse has been a focus of many research groups. The efforts to accomplish these goals have been carried out utilizing both the classical breeding and genetic engineering tools (Guo et al. 2003; Ruan et al. 2003; Li et al. 2004; Arioli, 2005; Ruan et al. 2005; Wilkins et al. 2005; Momtaz and Soliman, 2008). The over expression of G. hirsutum expansin gene has been reported for a fourfold increase in fiber yield that was cumulatively due to the increase in fiber length, number and fiber weight per seed. This study also reported the improvement of fiber quality traits by over-expressed expansin (Wilkins et al. 2005).

The C. procera fiber characteristics showed that this plant produces very fine, strong and long fibers as compared to other major sources of natural fibers (http://ccri.org.pk/publication/htm; Aziz, 2010). The shiny long single celled seed fibers of $C$. procera were explored for the expansin gene family. The isolation of four expansin homologs (CpEXPA1, CpEXPA2, CpEXPA3 and CpEXPA4) in elongating fibers suggested a strong role of this gene family in $C$. procera fiber development. The deduced amino acid sequence alignment with the well characterized expansins and predicted structural analysis showed that the derived amino acid sequences of the EXPA homologs consisted of two domains, i) $\mathrm{N}$ terminal domain and ii) C-terminal domain (Figure 4). In these genes a higher degree of conservation was observed in $\mathrm{N}$-terminal domain like other members of expansin gene family (Yennawar et al. 2006). The N-terminal domain has a distant homology to glycoside hydrolase family 45 (GH45)

Table 4. Amino acid sequence identity (\%) between C. procera expansin homologs in the coding region.

\begin{tabular}{lcccc}
\hline Sequence & CpEXPA1 & CpEXPA2 & CpEXPA3 & CpEXPA4 \\
CpEXPA1 & ---- & & & \\
CpEXPA2 & $63.03 \%$ & ----- & \\
CpEXPA3 & $70.14 \%$ & $63.87 \%$ & $-\cdots$ & \\
CpEXPA4 & $66.82 \%$ & $72.13 \%$ & $71.31 \%$ & ---- \\
\hline
\end{tabular}


proteins, most of which are fungal $\beta-1,4-D-e n d o g l u c a n a s e s$ (Davies et al. 1995; Xu et al.2001). The CpEXPAs have a number of conserved cysteins (Figure 4) just like the GH45, suggesting a conserved proteindomain (Fedorov et al. 1997). The His-Phe-Asp (HFD) motif found in the protein catalytic site was well conserved in CpEXPA1, CpEXPA2, CpEXPA3 and CpEXPA4 as observed in other members of EXPAs. Despite the presence of these conserved GH45motifs, no hydrolytic activity was detected for expansin polypeptides. Yennawar et al. (2006) described that expansins lacked a second aspartate that serves as the catalytic base in GH45 enzyme for its hydrolytic activity and the same can be observed in CpEXPA1, CpEXPA2, CpEXPA3 and CpEXPA4. The basic difference between EXPA and $E X P B$ is the presence of a large insertion and a nearby deletion in $\mathrm{N}$-terminal domain at either side of HFD motif, which was also observed in CpEXPA2, CpEXPA3 and CpEXPA4 (Figure 4).

Each predicted protein of the four expansin homologs included an $\mathrm{N}$-terminal signal sequence, which varied in length from 17 amino acids in CpEXPA4 to 26 residues in CpEXPA2. The signal peptide prediction results supplemented with protein targeting prediction (Table 4) indicated that all of the Expansin $A$ homologs produced secretory proteins. It means, that the nascent expansin proteins are processedby the secretory pathway that comprises of endoplasmic reticulum (ER), Golgi apparatus and cytoskeleton as reported earlier (Vitale and Denecke, 1999; Kessler and Neuhaus, 2003; Boutte et al. 2007). The presence of the signal anchor sites and the hydrophobic region comprising the transmembrane segment at the N-terminus (Table 4) suggested the insertion of this region as a loop in the central cavity of translocon (protein lined channel within the ER membrane). The absence of the ER retention signals (KDEL or HDEL as proposed by Denecke et al. 1992) in CpEXPA1, CpEXPA2, CpEXPA3 and CpEXPA4 and the secretory nature of these proteins suggested that these were targeted to the cell wall as assumed for members of Expansin A families in other plants (Cosgrove, 2000). The secretion of the C. procera fiber expansin is consistent with previous findings that expansin proteins are associated with plant cell walls and play key role in enlargement of the growing cell.

Phylogenetically, the isolated CpEXPAs were grouped along with various clades of EXPAs (Figure 4). CpEXPA1 is grouped with clade IV, which was previously described as a-Expansin subgroup A (Link and Cosgrove, 1998; Harmer et al. 2002; Gray-Mitsumun et al. 2004). This group is reported to have preferential expression in mature stems exhibiting secondary growth (Gray-Mitsumun et al. 2004). Although, the members of this group are involved in xylogenesis, they have also been reported to be involved in fruit ripening, (Reinhardt et al. 1998; Brummell et al. 1999a; Brummell et al. 1999b; Rose et al. 2000), cell wall disassembly during germination (Chen and Bradford, 2000) and the development of shoot and leaves (Im et al. 2000). However, some members of this group were also detected in multiple tissues analyzed from G. hirsutum and Glycine max (Harmer et al. 2002; Lee et al. 2003).

The phylogenetic relation (Figure 3) indicates that CpEXPA2 and GhEXPA4 fall within EXPA1 clade I formerly known as clade $C$ (Link and Cosgrove, 1998; Harmer et al. 2002). CpEXPA2 is grouped with GhEXPA3 and AtEXPA5, while CpEXPA4 is clustered along with AtEXPA1, AtEXPA10, AtEXPA15, GhEXPA6 and CsEXPA3 under the same node. This data indicates that CpEXPA2 is closer to the $G$. hirsutum EXPA3 while, CpEXPA4 is more related to the $G$. hirsutum EXPA6. The transcript profiling of CpEXPA2 and CpEXPA4 indicated the expression of these homologs in leave, stem and fibers, however, both the homologs were highly expressed in leave tissues, while CpEXPA4 also had a comparable expression in stem tissues (Figure 5). A similar heterologous expression pattern for other members of this clade has been reported (Shcherban et al. 1995; Brummell et al. 1999b; Harmer et al. 2002).

Thephylogenetic tree indicated that CpEXPA3 was placed with GhEXPA1 and GhEXPA2 in clade III (Figure 4), previously classified as clade B (Link and Cosgrove, 1998; Harmer et al. 2002; Cheema et al. 2006). The expression of GhEXPA1 and GhEXPA2 was observed in elongating cotton fibers, while the other tissues of $G$. hirsutum did not show their transcripts (Harmer et al. 2002). The CpEXPA3 also has significant expression in $C$. procera fibers but the maximum transcripts were detected in stem. This clade has been reported to contain many expansin genes which are detected in rapidly growing tissues (Shcherban et al. 1995; Catala et al. 2000; Shin et al. 2005). The CpEXPA3 may be an example of such genes in C. procera.

To determine the relative expression of $C p E X P A s$ in different $C$. procera tissues, the validated primers on the four homologs were selected for real time PCR studies. The expression profiling revealed that CpEXPA1 had the highest expression in stem tissues of $C$. procera but lesser transcription in fibers and leaves (Figure 5). This observation confirmed the involvement of clade IV expansins in vascular tissue development. An important observation related to the clade IV members is that none of them 
showed their expression in roots, though their transcripts have been reported in multiple tissues of the plants (Reinhardt et al. 1998; Brummell et al. 1999a; Im et al. 2000; Lee et al. 2003). The CpEXPA1 also showed the same expression pattern with negligible transcripts in roots (Figure 5). The specific functionality of this group may be related to the presence of unique conserved motifs at the $\mathrm{N}$-terminal

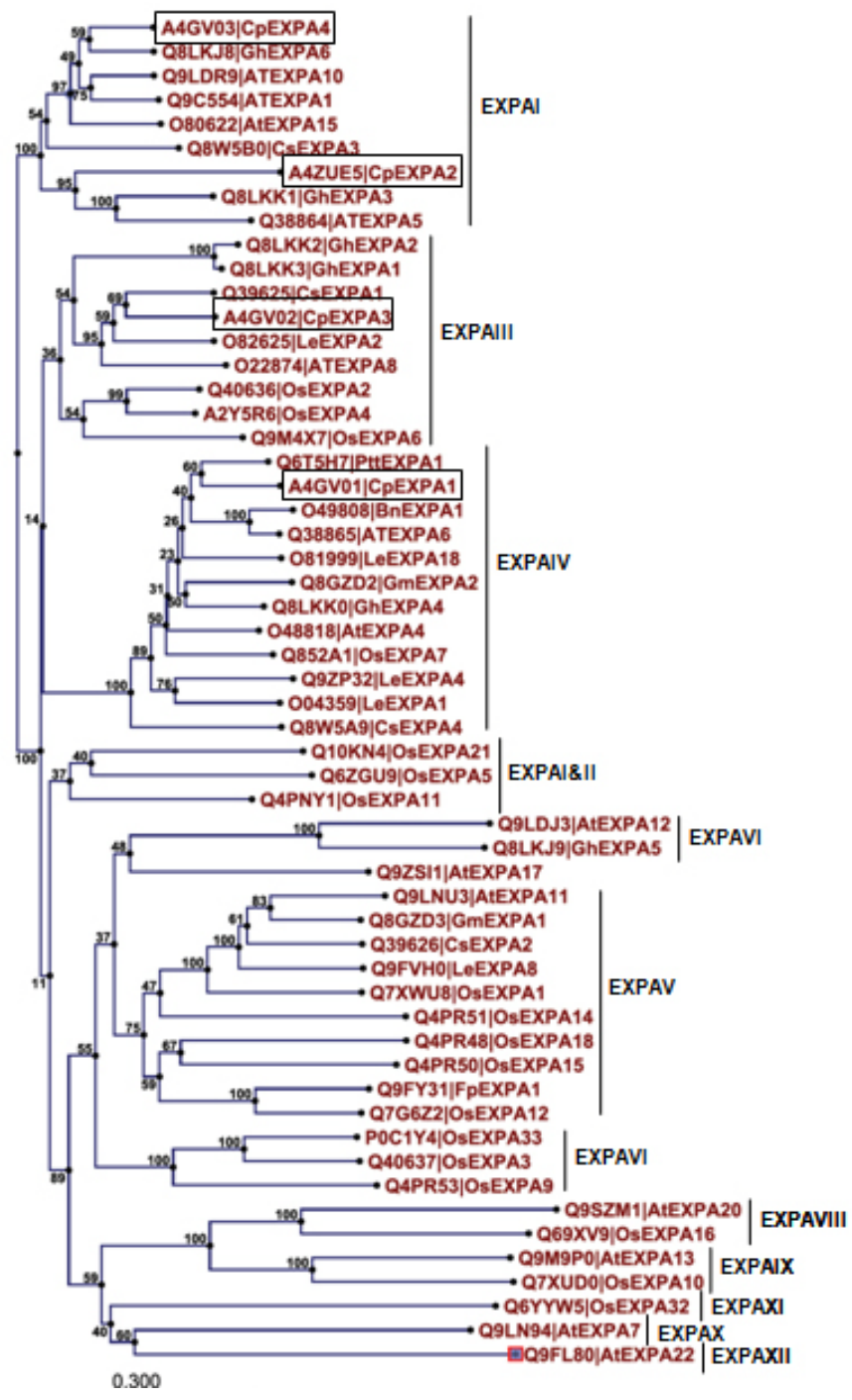

Fig. 4 Phylogenetic analysis of $C$. procera Expansin $A$ along with some other plant Expansin $A$ genes. Deduced amino acid sequences of full-length EXPA genes were used to construct the tree. EXPANSIN A gene source followed by Swiss ProTrEMBL accession numbers are as follows. Calotropis procera: CpEXPA1:A4GV01, CpEXPA2:A4ZUE5, CpEXPA3:A4GV02, CpEXPA4:A4GV03; Arabidopsis thaliana: AtEXPA1:Q9C554, AtEXPA10:Q9LDR9, AtEXPA15:080622, AtEXPA5:Q38864, AtEXPA8:022874, AtEXPA6:Q38865, AtEXPA4:048818, AtEXPA12:Q9LDJ3, AtEXPA17:Q9ZSI1, AtEXPA11:Q9LNU3, AtEXPA20:Q9SZM1, AtEXPA13:Q9M9P0, AtEXPA7:Q9LN94, AtEXPA22:Q9FL80. Gossypium hirsutum: GhEXPA1:Q8LKK3, GhEXPA2:Q8LKK2, GhEXPA3:Q8LKK1, GhEXPA4:Q8LKK0, GhEXPA5:Q8LKJ9, GhEXPA6:Q8LKJ8. Cucumis sativus: CsEXPA1:Q39625, CsEXPA2:Q39626, CsEXPA3:Q8W5B0, CsEXPA4:Q8W5A9, Oryza sativa: OsEXPA1:Q7XWU8, OsEXPA2:Q40636, OsEXPA3:Q40637, OsEXPA4:A2Y5R6, OsEXPA5:Q6ZGU9, OsEXPA6:Q9M4X7, OsEXPA7:Q852A1, OsEXPA9:Q4PR53, OsEXPA10:Q7XUD0, OsEXPA11:Q4PNY1, OsEXPA12:Q7G6Z2, OsEXPA14:Q4PR51, OsEXPA15:Q4PR50, OsEXPA16:Q69XV9, OsEXPA18:Q4PR48, OsEXPA21:Q10KN4, OsEXPA32:Q6YYWS, OsEXPA33:POC1Y4. Lycopersicon esculentum: LeEXPA1:O04359, LeEXPA2:O82625, LeEXPA4:Q9ZP32, LeEXPA8:Q9FVH0, LeEXPA18:081999. Populus tremula $x$ Populus tremuloides: PttEXPA1:Q6T5H7. Brassica napus: BnEXPA1:049808. Glycin max: GmEXPA1:Q8GZD3, GmEXPA2:Q8GZD2. Festuca pratensis: FPEXPA1:Q9FY31. 
and C-terminal region of the mature protein, RIPGV and KNFRV respectively. These sequences may endow some distinctive role in protein targeting to a specific component of cell wall or for interaction with other proteins and/or carbohydrates.

The transcriptome profiling of CpEXPA1, CpEXPA2, CpEXPA3 and CpEXPA4 depicted that these homologs are transcribed in different tissues (leaves, stem, root and fiber) and have variable expression. Interestingly, none of these identified expansins was fiber specific. This situation is different from $G$. hirsutum where two of the six $\alpha$-Expansins were identified to be fiber specific (Harmer et al. 2002; Cheema et al. 2006). Additionally, the identity level among these homologs is quite low (Table 4). The dilemma of the presence of these highly variable expansin isoforms in C. procera fibers and their exact role in the development of long shiny single celled fiber needs to be explored. The EXPAs have not been reported to be post transcriptionally regulated (Rose et al. 1997; Rose et al. 2000), therefore the transcript profiling of CpEXPAs could alternatively represent the expression of

(a)
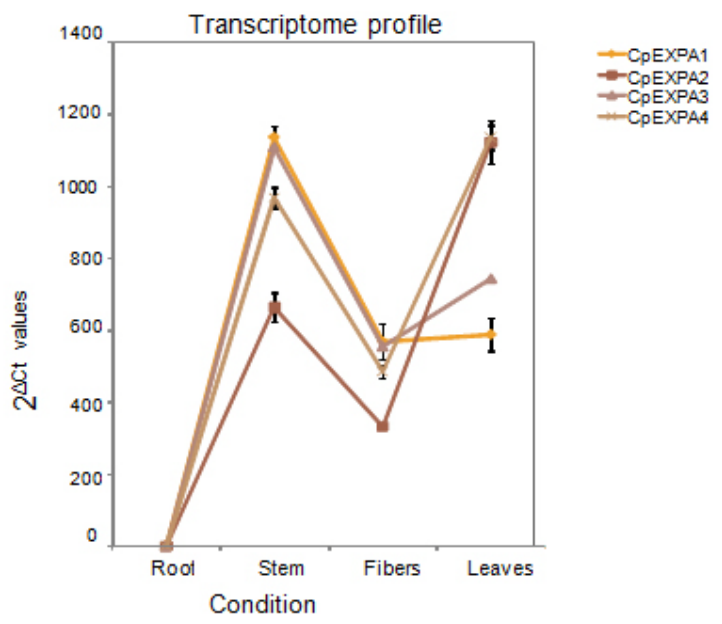

(b)

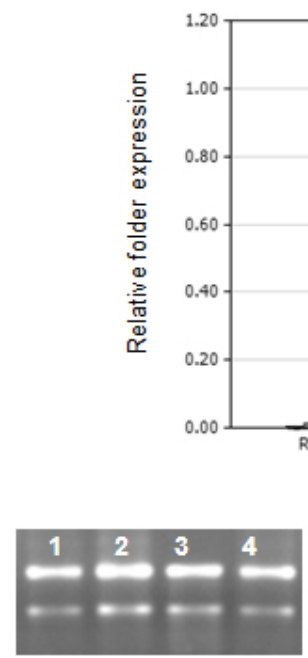

(c)

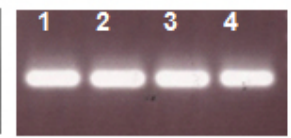

(d)

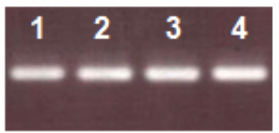

(e)

Fig. 5 Transcriptome profiling by Real-Time PCR. (a) Transcriptome profiling of CpEXPA1, CpEXPA2, CpEXPA3 and CpEXPA4 in C. procera roots, stem, fibers and leaves by real time RT-PCR using equalization of unit mass method. (b) Relative fold expression was determined by built-in software package of BioRad iQ5 thermal cycler. Relative quantification of transcriptome was determined by plotting the $2^{\Delta \mathrm{Ct}}$ values on $\mathrm{Y}$-axis against different tissues of $C$. procera. (c) Lane 1-4: $1 \%$ agarose gel containing equalized concentration of RNA isolated from root, stem, fiber and leave tissues respectively for their usage in the subsequent cDNA synthesis and real time PCR. (d) Lane 1-4: PCR amplification of 18srRNA gene in roots, stem, fibers and leaves respectively to equalize the amount of cDNA for normalization of the assay against unit mass. (e) Lane 1-4: PCR amplification of $\beta$-Tubulin gene in roots, stem, fibers and leaves respectively to equalize the amount of cDNA for normalization of the assay against unit mass. 
these genes. The expression of EXPAs has been observed in a number of species with their proved role in root elongation and development (Cho and Kende, 1998; Baluska et al. 2000; Cho and Cosgrove, 2002; Lee et al. 2003; Kam et al. 2005; Greenwood et al. 2006). The transcriptome profiling data of CpEXPA1, CpEXPA2, CpEXPA3 and CpEXPA4 indicated negligible expression of these genes in roots (Figure 5). This observation was in accordance with the previous report on characterizing and expression profiling of this gene family in rice (Shin et al. 2005). Our observation supports that $C$. procera might have some root specific expansins not detected in the fiber cDNA library.

Calotropis procera has been reported to be a habitant of deteriorated soil and grows well under extremes of draught conditions. The expansins coded by the plant may have evolved with respect to the harsh environmental stimuli and modifying the cellular configuration to meet the specific plant requirements. The expression of discrete expansin genes in stem and leaves may be related to the hardy nature of plant that produces softer stems and grows very well under difficult environmental conditions. The isolated expansin homologs represent useful natural resources to study their role in modifying plant traits including the fiber characteristics.

Financial support: This study was supported by a grant from Ministry of Food and Agriculture (MinFA). Partial support was provided by the PhD student grants from Higher Education Commission of Pakistan (HEC) for the PhD program carried out by Hafiza Masooma Naseer Cheema and Nadia Iqbal at NIBGE.

\section{REFERENCES}

ARIOLI, T. (2005). Genetic engineering for cotton fiber improvement. Bayer CropScience, vol. 58, no. 1, p. 140-150.

AZIZ, M.A. (2010). Introduction of new Egyptian cotton varieties. Cotton 247.com; Cotton International, Available from Internet at: http://cotton247.com/production/varieties/?storyid=1253. [Cited on July, 2010].

BALUŠKA, F.; SALAJ, J.; MATHUR, J.; BRAUN, M.; JASPER, F.; ŠAMAJ, J.; CHUA, N.H.; BARLOW, P.W. and VOLKMANN, D. (2000). Root hair formation: F-Actin-dependent tip growth is initiated by local assembly of profilin-supported F-Actin meshworks accumulated within expansin-enriched bulges. Developmental Biology, vol. 227, no. 2, p. 618-632. [CrossRef]

BENDTSEN, J.D.; NIELSEN, H.; VON HEIJNE, G. and BRUNAK, S. (2004). Improved prediction of signal peptides: SignalP 3.0. Journal of Molecular Biology, vol. 340, no. 4, p. 783-795. [CrossRef]

BOUTTE, Y.; VERNHETTES, S. and SATIAT-JEUNEMAITRE, B. (2007). Involvement of the cytoskeleton in the secretory pathway and plasma membrane organization of higher plant cells. Cell Biology International, vol. 31, no. 7, p. 649-654. [CrossRef]

BRUMMELL, D.A.; HARPSTER, M.H. and DUNSMUIR, P. (1999a). Differential expression of expansin gene family members during growth and ripening of tomato fruit. Plant Molecular Biology, vol. 39, no. 1, p. 161-169. [CrossRef]

BRUMMELL, D.A.; HARPSTER, M.H.; CIVELLO, P.M.; PALYS, J.M.; BENNETT, A.B. and DUNSMUIR, P. (1999b). Modification of expansin protein abundance in tomato fruit alters softening and cell wall polymer metabolism during ripening. Plant Cell, vol. 11, no. 11, p. 2203-2216. [CrossRef]

CAIUS, J.F. (1986). The medicinal and poisonous plants of India. Jodhpur, India, Scientific Publications, 528 p. ISBN 81-7233-326-9.

CATALÁ, C.; ROSE, J.K.C. and BENNETT, A.B. (2000). Auxin-regulated genes encoding cell wall-modifying proteins are expressed during early tomato fruit growth. Plant Physiology, vol. 122, no. 2, p. 527-534. [CrossRef]

CHEEMA, H.M.N.; BASHIR, A.; ZAFAR, Y. and MALIK, K.A. (2006). Identification of expansin variants in developing cotton fibers. In: International Egyptian-Jordanian Conference Under the Theme of Biotechnology and Sustainable Development: Current Status and Future Scenarios. $\left(1^{\text {st }}, 11^{\text {th }}-14^{\text {th }}\right.$ December, Cairo, Egypt). Abstracts. National Research Center, p. 210-211.

CHEN, F. and BRADFORD, K.J. (2000). Expression of an expansin is associated with endosperm weakening during tomato seed germination. Plant Physiology, vol. 124, no. 3, p. 1265-1274. [CrossRef]

CHO, H.T. and KENDE, H. (1998). Tissue localization of expansins in deepwater rice. The Plant Journal, September 1998, vol. 15, no. 6, p. 805-812. [CrossRef]

$\mathrm{CHO}, \mathrm{H} . T$. and COSGROVE, D.J. (2002). Regulation of root hair initiation and expansin gene expression in Arabidopsis. Plant Cell, vol. 14, no. 12, p. 3237-3253. [CrossRef]

COSGROVE, D.J.; BEDINGER, P. and DURACHKO, D.M. (1997). Group I allergens of grass pollen as cell wallloosening agents. Proceedings of the National Academy of Sciences of the United States of America, vol. 94 no. 12 , p. $6559-6564$.

COSGROVE, D.J. (2000). Loosening of plant cell walls by expansins. Nature, vol. 407, no. 6802, p. 321-326. [CrossRef]

COSGROVE, D.J.; LI, L.C.; CHO, H.T.; HOFFMANN-BENNING, S.; MOORE, R.C. and BLECKER, D. (2002). The growing world of expansins. Plant \& Cell Physiology, vol. 43, no. 12, p. 1436-1444. [CrossRef]

COSGROVE, D.J. (2005). Growth of the plant cell wall. Nature Reviews, Molecular Cell Biology, vol. 6, no. 11, p. 850-861. [CrossRef] 
DAVIES, G.J.; TOLLEY, S.P.; HENRISSAT, B.; HJORT, C. and SCHULEIN, M. (1995). Structures of oligosaccharide-bound forms of the endoglucanase $\mathrm{V}$ from Humicola insolens at 1.9 ANG. resolution. Biochemistry, vol. 34, no. 49, p. 16210-16220. [CrossRef]

DENECKE, J.; DE RYCKE, R. and BOTTERMAN, J. (1992). Plant and mammalian sorting signals for protein retention in the endoplasmic reticulum contain a conserved epitope. EMBO Journal, vol. 11, no. 6, p.23452355.

EISIKOWITCH, D. (1986). Morpho-ecological aspects on the pollination of Calotropis procera (Asclepiadaceae) in Israel. Plant Systematics and Evolution, vol. 152, no. 3-4, p. 185-194. [CrossRef]

FEDOROV, A.A.; BALL, T.; VALENTA, R. and ALMO, S.C. (1997). X-ray crystal structures of birch pollen profilin and $\mathrm{Phl}$ p 2. International Archives of Allergy and Immunology, vol. 113, no. 1-3, p. 109-113. [CrossRef]

GRAY-MITSUMUNE, M.; MELLEROWICZ, E.J.; ABE, H.; SCHRADER, J.; WINZÉLL, A.; STERKY, F.; BLOMQVIST, K.; MCQUEEN-MASON, S.; TEERI, T.T. and SUNDBERG, B. (2004). Expansins abundant in secondary xylem belong to subgroup $\mathrm{A}$ of the a-expansin gene family. Plant Physiology, vol. 135, no. 3, p. 1552-1564. [CrossRef]

GREENWOOD, M.S.; XU, F. and HUTCHISON, K.W. (2006). The role of auxin-induced peaks of $\alpha$-Expansin expression during lateral root primordium formation in Pinus taeda. Physiologia Plantarum, vol. 126, no. 2, p. 279-288. [CrossRef]

GUO, W.; SUN, J. and ZHANG, T. (2003). Gene cloning and molecular breeding to improve fiber qualities in cotton. Chinese Science Bulletin, vol. 48, no. 8, p. 709-717.

HARMER, S.E.; ORFORD, S.J. and TIMMIS, N.J.(2002). Characterisation of six a-Expansin genes in Gossypium hirsutum (upland cotton). Molecular Genetics and Genomics, vol. 268, no. 1, p. 1-9. [CrossRef]

IM, K.H.; COSGROVE, D.J. and JONES, A.M. (2000). Subcellular localization of expansin mRNA in xylem cells. Plant Physiology, vol. 123, no. 2, p. 463-470.

IQBAL, Z.; LATEEF, M.; JABBAR, A.; MUHAMMAD, G. and KHAN, M.N. (2005). Anthelmintic activity of Calotropis procera (Ait). Ait. F. flowers in sheep. Journal of Ethnopharmacology, vol. 102, no. 2, p. 256-261. [CrossRef]

JAIN, S.C.; SHARMA, R.; JAIN, R. and SHARMA, R.A. (1996). Antimicrobial activity of Calotropis procera. Fitoterapia, vol. 67, no. 3, p. 275-277.

KAM, M.J.; YUN, H.S.; KAUFMAN, P.B.; CHANG, S.C. and KIM, S.-K. (2005). Two expansins, EXP1 and EXPB2, are correlated with the growth and development of maize roots. Journal of Plant Biology, vol. 48, no. 3, p. 304-310. [CrossRef]

KENDE, H.; BRADFORD, K.; BRUMMELL, D.; CHO, H.T.; COSGROVE, D.J.; FLEMING, A.; GEHRING, C.; LEE, Y.; MCQUEEN-MASON, S.; ROSE, J. and VOESENEK, L. (2004). Nomenclature for members of the expansin superfamily of genes and proteins. Plant Molecular Biology, vol. 55, no. 3, p. 311-314. [CrossRef]

KESSLER, F. and NEUHAUS, J.M. (2003). Sorting activities in plant cells. Chimia International Journal for Chemistry, vol. 57 , no. 10 , p. $634-638$.

KWASNIEWSKI, M. and SZAREJKO, I. (2006). Molecular cloning and characterization of $\beta$-Expansin gene related to root hair formation in barley. Plant Physiology, vol. 141, no. 3, p. 1149-1158. [CrossRef]

LASSMANN, T. and SONNHAMMER, E.L.L. (2006). Kalign, Kalignvu and Mumsa: Web servers for multiple sequence alignment. Nucleic Acid Research, vol. 34, Web Server Issue, p. W596-W599. [CrossRef]

LEE, D.K.; AHN, J.H.; SONG, S.K.; CHOI, Y.D. and LEE, J.S. (2003). Expression of an expansin gene is correlated with root elongation in soybean. Plant Physiology, vol. 131, no. 3, p. 985-997. [CrossRef]

LI, L.C.; BEDINGER, P.A.; VOLK, C.; JONES, A.D. and COSGROVE, D.J. (2003). Purification and characterization of four $\beta$-Expansins (Zea m 1 isoforms) from maize pollen. Plant Physiology, vol. 132, no. 4, p. 2073-2085. [CrossRef]

LI, X.; WANG, X.D.; ZHAO, X. and DUTT, Y. (2004). Improvement of cotton fiber quality by transforming the acsA and acsB genes into Gossypium hirsutum L. by means of vacuum infiltration. Plant Cell Report, vol. 22, no. 9, p. 691-697. [CrossRef]

LI, Y.; DARLEY, C.P.; ONGARO, V.; FLEMING, A.; SCHIPPER, O.; BALDAUF, S.L. and MCQUEEN-MASON, S.J. (2002). Plant expansins are a complex multigene family with an ancient evolutionary origin. Plant Physiology, vol. 128 , no. 3, p. 854-864. [CrossRef]

LINK, B.M. and COSGROVE, D.J. (1998). Acid-growth response and a-Expansins in suspension cultures of Bright Yellow 2 tobacco. Plant Physiology, vol. 118, no. 3, p. 907-916. [CrossRef]

MARGA, F.; GRANDBOIS, M.; COSGROVE, D.J. and BASKIN, T.I. (2005). Cell wall extension results in the coordinate separation of parallel microfibrils: evidence from scanning electron microscopy and atomic force microscopy. Plant Journal, vol. 43, no. 2, p. 181-190. [CrossRef]

MCQUEEN-MASON, S.; DURACHKO, D.M. and COSGROVE, D.J. (1992). Two endogenous proteins that induce cell wall extension in plants. Plant Cell, vol. 4, no. 11, p. 1425-1433. [CrossRef]

MCQUEEN-MASON, S. and COSGROVE, D.J. (1994). Disruption of hydrogen bonding between wall polymers by proteins that induce plant wall extension. Proceedings of the National Academy of Sciences of the United States of America, vol. 91, no. 14, p. 6574-6578.

MCQUEEN-MASON, S.J. and COSGROVE, D.J. (1995). Expansin mode of action on cell walls. Analysis of wall hydrolysis, stress relaxation, and binding. Plant Physiology, vol. 107, no. 1, p. 87-100.

MOMTAZ, O.A. and SOLIMAN, K.M. (2008). Identification of genes associated with Egyptian cotton fiber development using combination of SSH, microarrays and real time RT-PCR. American-Eurasian Journal of Sustainable Agriculture, vol. 2, no. 1, p. 78-86.

MUEEN AHMED, K.K.; RANA, A.C. and DIXIT, V.K. (2005). Calotropis species (Ascelpediaceae) - A comprehensive review. Pharmacognosy Magazine, vol. 1, no. 2, p. 40-48.

OUDHIA, P. (1999a). Medicinal weeds in rice fields of Chhattisgarh (India). International Rice Research Note, vol. 24 , no. 1 , p. 40. 
OUDHIA, P. (1999b). Medicinal weeds in wheat fields of Chhattisgarh (India). Rachis (ICARDA), vol. 18, no. 1, p. 40-41.

RAGHAVAN, R.S. (1957). Chromosome numbers in Indian medicinal plants. Proceedings: Plant Sciences, vol. 45, no. 6, p. 294-298.

REINHARDT, D.; WITTWER, F.; MANDEL, T. and KUHLEMEIER, C. (1998). Localized upregulation of a new expansin gene predicts the site of leaf formation in the tomato meristem. The Plant Cell, vol. 10, no. 9, p. $1427-1437$.

ROSE, J.K.C.; LEE, H.H. and BENNETT, A.B. (1997). Expression of a divergent expansin gene is fruit- specific and ripening-regulated. Proceedings of the National Academy of Sciences of the United States of America, vol. 94, no. 11, p.5955-5960. [CrossRef]

ROSE, J.K.C.; COSGROVE, D.J.; ALBERShEIM, P.; DARVILL, A.G. and BENNETT, A.B. (2000). Detection of expansin proteins and activity during tomato fruit ontogeny. Plant Physiology, vol. 123, no. 4, p. 1583-1592. [CrossRef]

RUAN, Y.L.; LLEWELLYN, D.J. and FURBANK, R.T. (2003). Suppression of sucrose synthase gene expression represses cotton fiber cell initiation, elongation and seed development. Plant Cell, vol. 15, no. 4, p. 952-964. [CrossRef]

RUAN, Y.L.; LLEWELLYN, D.J.; FURBANK, R.T. and CHOUREY, P.S. (2005). The delayed initiation and slow elongation of fuzz-like short fibres in relation to altered patterns of sucrose synthase expression and plasmodesmata gating in a lintless mutant of cotton. Journal of Experimental Botany, vol. 56, no. 413, p. 977984. [CrossRef]

SABIRZHANOVA, I.B.; SABIRZHANOV, B.E.; CHEMERIS, A.V.; VESELOV, D.S. and KUDOYAROVA, G.R. (2005). Fast changes in expression of expansin gene and leaf extensibility in osmotically stressed maize plants. Plant Physiology and Biochemistry, vol. 43, no. 4, p. 419-422. [CrossRef]

SAKTHIVEL, J.C.; MUKHOPADHYAY, S. and PALANISAMY, N.K. (2005). Some studies on Mudar fibers. Journal of Industrial Textile, vol. 35, no. 1, p. 63-76. [CrossRef]

SAMPEDRO, J. and COSGROVE, D.J. (2005). The expansin superfamily. Genome Biology, vol. 6, no. 12, p. 242. [CrossRef]

SCHIPPER, O.; SCHAEFER, D.; RESKI, R. and FLEMING, A. (2002). Expansins in the bryophyte Physcomitrella patens. Plant Molecular Biology, vol. 50, no. 4-5, p. 789-802. [CrossRef]

SHCHERBAN, T.Y.; SHI, J.; DURACHKO, D.M.; GUILTINAN, M.J.; MCQUEEN-MASON, S.J.; SHIEH, M. and COSGROVE, D.J. (1995). Molecular cloning and sequence analysis of expansins - a highly conserved, multigene family of proteins that mediate cell wall extension in plants. Proceedings of the National Academy of Sciences of the United States of America, vol. 92, no. 20, p. 9245-9249. [CrossRef]

SHIN, J.H.; JEONG, D.H.; PARK, M.C. and AN, G. (2005). Characterization and transcriptional expression of the $\alpha-$ Expansin gene family in rice. Molecules and Cells, vol. 20, no. 2, p. 210-218.

VARSHNEY, A.C. and BHOI, K.L. (1988). Cloth from bast fibre of the Calotropis procera (Aak) plant. Biological Wastes, vol. 26, no. 3, p. 229-232. [CrossRef]

VITALE, A. and DENECKE, J. (1999). The endoplasmic reticulum - gateway of the secretory pathway. Plant Cell, vol. 11, no. 4, p. 615-628. [CrossRef]

VON HEIJNE, G. (1986). A new method for predicting signal sequence cleavage sites. Nucleic Acids Research, vol. 14, no. 11, p. 4683-4690. [CrossRef]

WILKINS, T.A.; ARPART, A. and SICKLER, B. (2005). Cotton fiber genomics: Developmental mechanisms. Bayer CropScience Journal, vol. 58, p. 119-139.

WU, Y.; MEELEY, R.B. and COSGROVE, D.J. (2001). Analysis and expression of the $\alpha$-Expansin and $\beta$-Expansin gene families in maize. Plant Physiology, vol. 126, no. 1, p. 222-232. [CrossRef]

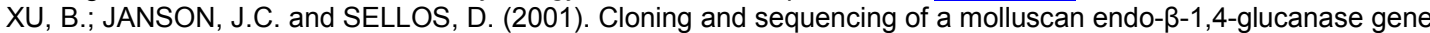
from the blue mussel, Mytilus edulis. European Journal of Biochemistry, vol. 268, no. 13, p. 3718-3727. [CrossRef]

YENNAWAR, N.H.; LI, L.C.; DUDZINSKI, D.M.; TABUCHI, A. and COSGROVE, D.J. (2006). Inaugural article: Crystal structure and activities of EXPB ${ }_{1}$ (Zea m 1), a $\beta$-Expansin and group-1 pollen allergen from maize. Proceedings of the National Academy of Sciences, vol. 103, no. 40, p. 14664-14671. [CrossRef]

\section{How to cite this article:}

CHEEMA, H.M.N.; BASHIR, A.; KHATOON, A.; IQBAL, N.; ZAFAR, Y.; MALIK, K.A. ( 2010). Molecular characterization and transcriptome profiling of expansin genes isolated from Calotropis procera fibers. Electronic Journal of Biotechnology, vol. 13, no. 6. http://dx.doi.org/10.2225/vol13-issue6-fulltext-7 